\title{
Dyslipidaemia, statins and rheumatoid arthritis
}

\section{$\mathcal{V}$ Michael T Nurmohamed, ${ }^{1,2,3}$ Ben A C Dijkmans ${ }^{1,3}$ EDITOR'S \\ CHOICE}

Cardiovascular morbidity and mortality are enhanced in rheumatoid arthritis (RA) and there is increasing evidence that this is due to the inflammatory process as well as to an increased prevalence of traditional cardiovascular risk factors, such as dyslipidaemia. ${ }^{1-3}$

\section{DYSLIPIDAEMIA IN ESTABLISHED AND FUTURE PATIENTS WITH RA}

Several investigators have indeed demonstrated dyslipidaemia, defined as higher total cholesterol and/or triglycerides and/or lower high-density lipoprotein (HDL) cholesterol levels in comparison to control subjects, in RA and this appears to be the consequence of systemic release of inflammatory cytokines such as tumour necrosis factor (TNF) $\alpha$, interleukin (IL)1 and IL6, leading to a proatherogenic state with insulin resistance, endothelial cell activation and hypercoagulation as other consequences.

The dyslipidaemia in RA is dependent on disease activity, ie, a higher disease activity is associated with lower total cholesterol levels and even more depressed HDL cholesterol levels, leading to a higher (ie, unfavourable) atherogenic index. ${ }^{4}$

Moreover, it appears that dyslipidaemia is already present in early RA and the question arises whether or not this phenomenon starts in the preclinical phase of RA. Hence, we investigated the lipid profile over time and its relationship with inflammation and serological markers, in subjects who later developed RA. ${ }^{5}$ The lipid profile was determined in 1078 serial blood bank samples, of 79 blood donors who later developed RA. These samples were compared with 1071 control samples of unselected blood donors, matched for age and sex. The samples of future patients with RA displayed, on average, $4 \%$ higher total cholesterol, 9\%

\footnotetext{
${ }^{1}$ Department of Internal Medicine, VU University Medical Centre, Amsterdam, The Netherlands; ${ }^{2}$ Department of Rheumatology, VU University Medical Centre,

Amsterdam, The Netherlands; ${ }^{3}$ VU University Medical Centre, Jan van Breemen Institute, Amsterdam, The Netherlands

Correspondence to: Dr Michael T Nurmohamed, Departments of Internal Medicine and Rheumatology, VU University Medical Centre, PO Box 7057, Amsterdam, The Netherlands; mt.nurmohamed@vumc.nl
}

lower HDL cholesterol and 17\% higher triglyceride levels compared to matched controls ( $p \leqslant 0.05)$, at least 10 years before the onset of RA symptoms. Although the differences in the various lipid values were small they may have clinical relevance, in the light of results from other studies. For instance, in a placebo-controlled study with fibrates, the differences of the lipid values between the active treatment and the placebo group were similar to the observed differences in our study and the individuals treated with fibrates had ultimately a more than $20 \%$ risk reduction for cardiovascular disease. ${ }^{6}$

\section{HDL CHOLESTEROL}

As the observed differences in lipid levels between future patients with RA and the control subjects were only partially explained by the differences in C-reactive protein (CRP) levels, alternative explanations are required. A tempting idea is that a (marginally) deteriorated lipid profile may render a person more susceptible to inflammation or inflammatory diseases. In other words, one or more of the examined lipids could have a regulatory effect on inflammation. It is well known that contact-mediated activation of monocytes by stimulated $\mathrm{T}$ lymphocytes is important for the production of TNF $\alpha$ and interleukin 1 in RA. Hyka et al demonstrated, in an experimental model, the ability of apolipoprotein A-I (apo A-I), the protein part of $\mathrm{HDL}$ cholesterol, to inhibit this inflammatory response. ${ }^{7}$ It appeared that apo A-I hampers the binding of $\mathrm{T}$ lymphocytes to monocytes with subsequent abolishing of TNF $\alpha$ and IL1 production. In addition to the contact mediated inhibition of monocytes, HDL cholesterol inhibits the expression of vascular cell adhesion molecule 1 (VCAM-1) and intercellular adhesion molecule 1 (ICAM-1) in TNF $\alpha$ stimulated human cells. ${ }^{8}$

Altogether these data indicate an active modulating role of lipids in inflammation. It is important to realise that inflammation itself might alter the properties of HDL cholesterol Atherosclerosis starts when low-density lipoprotein (LDL) cholesterol infiltrates the artery wall and is oxidised by reactive oxygen species to oxidised LDL cholesterol (ox-LDL cholesterol). Ox-LDL cholesterol leads to phospholipid release, activating endothelial cells, thereby initiating an inflammatory process that leads to the formation of foam cells and subsequent fatty streaks. Normal HDL cholesterol exerts its antiatherogenic role by protecting LDL cholesterol from oxidation, ${ }^{9}$ in addition to the inhibition of the expression of adhesion molecules and its role in the reverse cholesterol transport. This anti-inflammatory HDL cholesterol can be distinguished from the so-called proinflammatory HDL cholesterol which does not have these properties and actually may promote inflammation. ${ }^{10}$ Recently, McMahon and colleagues showed that proinflammatory HDL cholesterol was detected more often in patients with RA $(n=48)$ than in control subjects, $(n=72)$ (ie, $20 \%$ vs $4 \%$, respectively). ${ }^{11}$ This impairment of the ability of HDL cholesterol to prevent oxidation of LDL cholesterol might predispose RA to development of cardiovascular disease and effective antirheumatic treatment might restore the functional capacities of HDL cholesterol. ${ }^{12}$

\section{LIPID LOWERING AGENTS AND (THE DEVELOPMENT OF) RA}

In view of the above-mentioned interplay between lipids and inflammation, it is of interest to study the effect of lipid modulation by cholesterol lowering agents, particularly statins, in inflammatory situations. It has been demonstrated that several statins significantly increase HDL cholesterol and apo A-I through upregulation of apo A-I synthesis, in vitro as well as in vivo. ${ }^{13}{ }^{14}$ As a consequence, this could lead to modulation of inflammatory processes.

A pivotal feature of RA is inflammation associated bone destruction and as recent data indicate that statins are potent inducers of bone morphogenic protein and osteoclastogenesis inhibitors, ${ }^{15}$ Funk et al investigated their potential bone protective properties. ${ }^{16}$ In an experimental rat arthritis model, simvastatin was given to female Lewis rats 4 days before or 8 days after induction of arthritis and simvastatin prevented early and late joint inflammation and this was associated with a decrease in articular macrophage influx. Moreover, simvastatin inhibited periarticular bone destruction occurring late in the course of disease and preserved periarticular bone mineral density. These results suggest that statins may be therapeutically useful in preserving periarticular bone in RA joints 
via suppression of inflammation-induced bone resorption.

These properties of statins warranted further clinical investigation and after a small clinical investigation with simvastatin revealed an impressive $50 \%$ reduction of disease activity in 9 out of 10 patients, ${ }^{17}$ a double-blind placebo-controlled trial with another statin (atorvastatin) in 116 patients with RA was conducted. ${ }^{18}$ These patients were either treated with atorvastatin $40 \mathrm{mg}$ or placebo for 6 months, in addition to their disease-modifying antirheumatic drug (DMARD) therapy. At 6 months, there was a modest, but significant, improvement of the disease activity $(0.5$ point on the disease activity 28 score) in the atorvastatin group compared to the placebo group with a CRP and erythrocyte sedimentation rate decline of $50 \%$ and $28 \%$, respectively. Obviously, there is now a need for larger investigations with longer follow-up, that investigate if, and to what extent (and at what dose), statins can prevent the bone destruction in RA.

Altogether, lipids and alteration of the lipid profile by statins have the potential to modulate inflammatory features of RA and a tempting hypothesis is that they therefore could also have a role in the development of RA or other inflammatory disorders. For instance, the West of Scotland Coronary Prevention Study (WOSCOPS) revealed 30\% less new onset diabetes in the men treated with pravastatin vs the placebo-treated persons. ${ }^{19}$ One of the explanations may be that pravastatin lowers the levels of IL6 and TNF $\alpha$ resulting in a decreased lipolysis in adipose tissue and less insulin resistance, and recently it was demonstrated that pravastatin additionally increases the levels of adiponectin, an adipose tissue derived cytokine with anti-atherosclerotic and insulin-sensitising functions. ${ }^{20}$

Likewise a lower incidence of RA would also be expected in "normal" subjects receiving lipid lowering drugs vs those who did not, in controlled clinical trials investigating these drugs. Unfortunately, this topic has not yet been addressed in such trials. However, a recent epidemiological investigation has shed some light on this topic. ${ }^{21}$

The General Practice Research Database was used in this nested case-control study and this database was founded in 1987 and presently encompasses 5 million UK residents who are representative of the UK population. The present study period was 10 years (until 31 December 2001) and three groups of subjects aged 40 to 89 years were identified from the database: (a) patients exposed to a statin or other lipid lowering drugs, (b) subjects with a hyperlipidaemia diagnosis with no lipidlowering drugs and (c) a random sample of 25000 persons with no hyperlipidaemia nor prescriptions for lipid-lowering drugs.

From the database population incident cases of RA were identified and up to four controls per case, matched on age, sex, general practice, years of history in the database and index date were selected. Conditional logistic regression techniques were used to investigate the effects of hyperlipidaemia and statins on the development of RA. A total of 313 incident cases of RA were identified and matched with 1252 controls. RA cases had hyperlipidaemia more often than controls, and there was a $30 \%$ risk increase for RA in those persons with untreated hyperlipidaemia (odds ratio (OR) 1.33, 95\% CI 0.95 to 1.86$)$ and persons with no hyperlipidaemia had a $30 \%$ risk decrease for RA (OR 0.68, 95\% CI 0.50 to 0.91).

Compared to persons with hyperlipidaemia but no statin, current statin use reduced the risk for the development of RA with $30 \%$ (those with statin and hyperlipidaemia) to $40 \%$ (those with statin without hyperlipidaemia, albeit that this reduction did not reach statistical significance). Subgroup analyses revealed no important differences among the three most commonly prescribed statins (ie, simvastatin, pravastatin and atorvastatin). Remarkably, lipid lowering drugs other that statins were not associated with a decreased risk. This might be due to too low a patient number treated with these drugs, a too limited effect on the lipid profile or the absence of antiinflammatory properties of these drugs.

This investigation reveals two important points: (1) the association between hyperlipidaemia and the development of RA, which is in line with the findings of van Halm et $a l^{5}$ and (2) that statins are protective against development of RA in those with hyperlipidaemia.

Future studies will have to address whether or not statins really protect against the development of RA or if they only delay disease onset.

\section{CONCLUSIONS}

There is a growing body of evidence indicating relationships between dyslipidaemia and (the development) of RA. At least 10 years before disease onset there is a disturbed lipid profile and future studies should elucidate if this dyslipidaemia renders a person more susceptible for the development of RA, through proinflammatory mechanisms or if there is a coupled genetic or socioeconomic background. As a consequence targeting dyslipidaemia may influence inflammation and the development of inflammatory disorders. Indeed, the literature suggests that statins may have a moderate diseasemodifying effect in RA and this is, at least partly, independent from their cholesterol lowering properties and ascribed to their pleiotropic anti-inflammatory actions. Very recently, it appeared that these drugs might also prevent (or retard) the development of RA.

The challenge is obviously to translate the (presumed) RA preventing effect of statins into clinical practice. Over the past few years there has been a continuing debate as to whether or not a polypill that combines aspirin, a statin for lowering cholesterol and an angioconverting enzyme inhibitor with a thiazide to lower blood pressure should be given to all persons with an increased cardiovascular risk (eg, elderly persons). ${ }^{22}{ }^{23}$ Presently, the first international placebo-controlled trial has been initiated, ${ }^{24}$ which will be followed by large-scale placebo-controlled investigations when the first results demonstrate that the polypill is tolerable and efficacious. Perhaps, the combination of all these trials might show a favourable effect of statin/polypill on the incidence of inflammatory diseases such as RA, albeit that the number of incident cases might still be too low.

The clinical use of statins is more realistic in the context of cardiovascular risk management in RA. When lipid lowering therapy is necessary, statins are preferred over other lipid lowering drugs, as they not only target dyslipidaemia but also have anti-inflammatory properties and this is important as nowadays cardiovascular risk management in RA should be targeted at the inflammatory process as well as the cardiovascular risk factors. ${ }^{25}$

Competing interests: None declared.

Accepted 11 January 2009

Ann Rheum Dis 2009;68:453-455.

doi:10.1136/ard.2008.104497

\section{REFERENCES}

1. Solomon DH, Greenberg J, Reed G, Setoguchi S, TsaoP, Kremer J. Cardiovascular risk among patients with RA in CORRONA: comparing the explanatory value of traditional cardiovascular risk factors with RA risk factors. Ann Rheum Dis 2008;67(Suppl 2):482.

2. van Halm VP, Peters MJ, Voskuyl $A E$, Boers $M$, Lems WF, Visser $M$, et al. Rheumatoid arthritis versus diabetes as a risk factor for cardiovascular disease, a cross sectional study. The CARRE Investigation. Ann Rheum Dis. Published Online First: 12 August 2008. doi:10.1136/ard.2008.094151

3. Peters MJL, van Halm VP, Voskuyl AE, Boers M, Lems WF, Visser $M$, et al. Rheumatoid arthritis as important independent risk factor for incident 
cardiovascular disease. Arthritis Rheum 2008;58(Suppl): S421-2.

4. Boers M, Nurmohamed MT, Doelman CJ, Lard LR, Verhoeven AC, Voskuyl AE, et al. Influence of glucocorticoids and disease activity on total and high density lipoprotein cholesterol in patients with rheumatoid arthritis. Ann Rheum Dis 2003;62:842-5.

5. van Halm VP, Nielen MM, Nurmohamed MT, van Schaardenburg D, Reesink HW, Voskuyl AE, et al. Lipids and inflammation - serial measurements of the lipid profile of blood donors who later developed rheumatoid arthritis. Ann Rheum Dis 2007;66:184-8.

6. Rubins HB, Robins SJ, Collins D, Fye CL, Anderson JW, Elam MB, et al. Gemfibrozil for the secondary prevention of coronary heart disease in men with low levels of high-density lipoprotein cholesterol. Veterans Affairs High-Density Lipoprotein Cholesterol Intervention Trial Study Group. N Engl J Med 1999;341:410-8

7. Hyka N, Dayer JM, Modoux C, Kono T, Edwards CK, Roux-Lombard $\mathrm{P}$, et al. Apolipoprotein A-I inhibits the production of interleukin $1 \beta$ and tumor necrosis factor- $\alpha$ by blocking contact mediated activation of monocytes by T-lymphocytes. Blood 2001;97:2381-9.

8. Ashby DT, Rye KA, Clay, MA, Vadas MA, Gamble JR, Barter PJ. Factors influencing the ability of HDL to inhibit expression of vascular cell adhesion molecule-1 in endothelial cells. Arterio Thromb Vasc Biol 1998;18:1450-5.

9. Ansell BJ, Navab M, Watson KE, Fonarow GC, Fogelman AM. Anti-inflammatory properties of HDL. Rev Endocr Metab Disord 2004;5:351-8.

10. Navab M, Hama SY, Hough GP, Subbanagounder G, Reddy ST, Fogelman AM. A cell-free assay for detecting $\mathrm{HDL}$ that is dysfunctional in preventing the formation of or inactivating oxidized phospholipids. $J$ Lipid Res 2001;42:1308-17.

11. McMahon M, Grossman J, FitzGerald J, Dahlin-Lee E, Wallace DJ, Thong BY. Proinflammatory highdensity lipoprotein as a biomarker for atherosclerosis in patients with systemic lupus erythematosus and rheumatoid arthritis. Arthritis Rheum 2006;54:2541-9.

12. Charles-Schoeman C, Lee YY, Furst DE, Paukus HE, McMahon M, Fogelman AM, et al. Methotrexate improves HDL's anti-inflammatory properties in patients with systemic rheumatic disease. Arthritis Rheum 2008;58(Suppl): S271.

13. Martin G, Duez H, Blanquart C, Berezowski V, Poulain $P$, Fruchart JC, et al. Statin-induced inhibition of the Rho-signaling pathway activates PPAR $\alpha$ and induces HDL apoA-I. J Clin. Invest 2007;107:1423-32.

14. Charles-Schoeman C, Khanna D, Furst DE McMahon M, Reddy ST, Fogelman AM, et al. Effects of high-dose atorvastatin on antiinflammatory properties of high density lipoprotein in patients with rheumatoid arthritis: a pilot study. J Rheumatol 2007:34:1459-64.

15. Mundy G, Garrett R, Harris S, Chan J, Chen D, Rossini $G$, et al. Stimulation of bone formation in vitro and in rodents by statins. Science 1999;286:1946-9.

16. Funk JL, Chen J, Downey KJ, Clark RA. Bone protective effect of simvastatin in experimental arthritis. J Rheumatol 2008:35:1083-91.

17. Abud-Mendoza C, de la Fuente H, Cuevas-Orta E, Baranda L, Cruz-Rizo J, González-Amaro R Therapy with statins in patients with refractory rheumatic diseases: a preliminary study. Lupus 2003;12:607-11.
18. McCarey DW, Mclnnes IB, Madhok R, Hampson R Scherbakov 0 , Ford I, et al. Trial of Atorvastatin in Rheumatoid Arthritis (TARA): double-blind, randomised placebo-controlled trial. Lancet 2004;363:2015-21.

19. Freeman DJ, Norrie J, Sattar N, Neely RD, Cobbe SM, Ford I, et al. Pravastatin and the development of diabetes mellitus: evidence for a protective treatment effect in the West of Scotland Coronary Prevention Study. Circulation 2001;103:357-62.

20. Takagi T, Matsuda M, Abe M, Kobayashi $\mathrm{H}$, Fukuhara A, Komuro R, et al. Effect of pravastatin on the development of diabetes and adiponectin production. Atherosclerosis 2008:196:114-21.

21. Jick SS, Choi H, Li L, Mclnnes IB, Sattar N Hyperlipidemia, statin use and the risk of developing rheumatoid arthritis. Ann Rheum Dis. Published Online First: 28 July 2008. doi:10.1136/ard.2008.091967

22. Wald NJ, Law MR. A strategy to reduce cardiovascular disease by more than $80 \%$. BMJ 2003:326:1419-24.

23. Watts G. What happened to the polypill? BMJ 2008;337:a1822.

24. Clinical Trials Unit, Department of Medicine, Auckland University. PILL pilot: Programme to Improve Life and Longevity. http://www.ctru. auckland.ac.nz/content/view/37/35/ (accessed 24 December 2008).

25. Peters MJ, Symmons DPM, McCarey DW. Nurmohamed MT. Cardiovascular risk management in patients with rheumatoid arthritis and other types of inflammatory arthritis - a EULAR task force. Arthritis Rheum 2008;58(Suppl):S421. 